\title{
A case series of patients, including a consultant rhinologist, who all experienced a loss of smell associated with confirmed or suspected COVID-19*
}

\section{David E.J. Whitehead', Christine Kelly², N. Ahmad'}

'Department of Otorhinolaryngology, James Cook University Hospital, Middlesbrough, United Kingdom

2 AbScent, 14 London Road, Andover, Hampshire, United Kingdom
Rhinology Online, Vol 3: 67 - 72, 2020

http://doi.org/10.4193/RHINOL/20.027

*Received for publication:

April 10, 2020

Accepted: May 22, 2020

Published: May 28, 2020

\begin{abstract}
Background: Non-invasive detection of carriers of COVID-19 virus remains elusive. A decrease in sense of smell appears to be a potential marker of the disease. However, it is not the most frequently reported complaint and there may be more novel early markers of disease.
\end{abstract}

Methodology: We present a case series of patients, including a consultant rhinologist who all experienced a loss of smell associated with confirmed or suspected COVID-19.

Results: A consultant rhinologist presented with a delayed sudden onset anosmia, four days after testing positive for coronavirus whilst also exhibiting evidence of autonomic dysfunction prior to rRT-PCR diagnosis and during the time period during which smell suddenly deteriorated. Sudden loss of smell can occur within a 3-hour window and a transient increase in SNOT-22 score was also noted at the time of loss.

Conclusions: Transient hyposmia or anosmia appear to be an early warning sign or marker symptom associated with COVID-19. Smell can be lost rapidly but appears to recover for many. For others a variety of novel treatments exist. There may be more sensitive or specific signs associated with the disease.

Key words: anosmia, autonomic dysfunction, autonomic nervous system, coronavirus, COVID-19, heart rate variability, hyposmia, olfactory loss, smell training, SNOT-22

\section{Introduction}

Case report 1

A 49-year old Rhinologist (the first author) developed a mild cough, rigors and a pyrexia of $40.2^{\circ} \mathrm{C}$ following a week on-call operating on multiple suspected COVID-19 patients (wearing Personal Protective Equipment, PPE). Two days after becoming symptomatic real-time reverse transcription polymerase chain reaction ( $r R T-P C R)$ testing confirmed he was positive for COVID-19. Four days after noticing symptoms he developed a mild nasal congestion and rhinorrhoea.

His sense of smell was normal, and he continued to improve until 7 days after developing his first symptoms when at 15:35hrs he noticed a headache and hyposmia ${ }^{(1)}$. At 17:03hrs $90 \%$ of his sense of smell had been subjectively lost and congestion confined to high up in the nasal vault (cavity) was reported as a symptom ${ }^{(2)}$, with no turbinate congestion. At 18:30hrs his temperature rose to $38.6^{\circ} \mathrm{C}$ and complete anosmia was noted. There was some associated sneezing and his NOSE score at that point was 3 and his SNOT-22 ${ }^{(3)}$ score was 33.

At 23:20hrs he developed complete nasal blockage on the left side and rhinorrhoea affecting the right nasal cavity. He developed bilateral ear congestion and a burning sensation in the postnasal space similar to that experienced by the author many decades before when he had adenovirus. 
On day 8 at 07:44hrs he could barely smell lemon Verbena in hand soap and grapefruit in facial wash. Subjectively, he had lost $98 \%$ of his sense of smell. At $12: 16 \mathrm{hrs}, 5 \%$ of his smell had returned. His NOSE score had dropped to 1 and the SNOT-22 score had dropped to 8 . At 22:24hrs the NOSE score was 0 and SNOT-22 was 9.

By day 9 his NOSE score was $=0$ and SNOT-22 $=12^{(4)}$. A heat map of all SNOT-22 scores is shown in Figure 1.

He noticed an increased tolerance to Capsaicin in chilli peppers and freshly ground black pepper. Its effect was reduced and confined only to the oral cavity.

A trial of $0.1 \%$ Xylometazoline Hydrochloride nasal spray had no effect on subjective smell perception.

Four days following the sudden onset of anosmia, his smell had partially recovered with an UPSIT ${ }^{(5)}$ score of 27 (which is moderate microsmia adjusted for age). Six days after complete anosmia a retest using the UPSIT showed a score of 27 . Six weeks following anosmia UPSIT score was 39 and within the 90th percentile adjusted for the age.

\section{Case report 2}

A 50-year old female had a minor procedure and the next day she noticed symptoms of mild rhinitis and her husband became unwell with symptoms of coronavirus. The following day she noted a sudden loss of smell and a very tender right eye, followed 2 days later by a pyrexia of $38.4^{\circ} \mathrm{C}$. She felt lethargic, developed a cough at the end of the first week, as well as nasal congestion, rhinorrhoea and heart palpitations. Seven days later subjectively $85-90 \%$ of her sense of smell returned and she continues to experience minor acute rhinosinusitis.

\section{Case report 3}

A 50-year old female woke with a unilateral "migraine," nausea and complete anosmia but no loss of taste sensation, instead noticing increased sensitivity to salt and vinegar. This was the worst migraine she had ever experienced and her symptoms worsened until day 3 when she also experienced rigors. She remained in bed and could not eat without vomiting. She did not experience nasal congestion but felt lightheaded on day 4 and 5 . Her sense of smell started to return on day 6 .

\section{Case report 4}

A 21-year old female student incidentally noticed a complete acute loss of sense of smell while preparing a barbeque. Two days later she developed headache, nasal congestion, chest tightness, a mild cough, as well as fatigue and light-headedness. Her nasal congestion continued for 2 weeks and the headache for 10 days. She subjectively felt that $95 \%$ of her sense of smell had been lost. She was only able to partially smell (in a distorted fashion) washing up liquid. She could feel the effect of spices/ Capsaicin and salt in her mouth but lost her ability to detect sweet foods or liquids.

\section{Other evidence of infection}

Confirmation of active COVID-19 virus depends on a positive real-time reverse transcription polymerase chain reaction (rRT$\mathrm{PCR}$ ) test. Technical issues related to obtaining representative viral sampling within 72 hours of onset of symptoms can lead to false negatives. Alternative machine learning methods may provide a non-invasive and less time critical method, a "synthetic test" of predicting who may have COVID-19. ZOE Global Ltd a nutritional science company and King's College London data scientists have created a model to predict COVID-19 in the UK. Of people who were definitely infected with coronavirus, 59\% reported losing their sense of smell or taste. Of people not yet tested, the model predicted $13 \%$ are likely to be infected.

Heart rate variability (HRV) represents a non-invasive method of looking at autonomic activity and physiological status of the human body. Several studies ${ }^{(6-8)}$ have examined the usefulness of HRV for the early diagnosis and prognosis of viral infections.

Wearable technology that continuously measures HRV is widely available from a number of sources (Firstbeat Technologies Oy, Yliopistonkatu 28 A, Fl-40100 Jyväskylä, Finland; Garmin Ltd. Liberty House Hounsdown Business Park, Southampton SO40 9LR, UK; WHOOP, Inc., 1325 Boylston Street, Suite 401, Boston, MA 02215, USA and EliteHRV). The first author recorded HRV and 'stress levels' during the onset of first symptom and prior to rRTPCR testing. When smell was lost seen in Figure 2, a significant increase in stress levels (due to a drop in HRV) compared to the patient's normal stress levels of between 19-25 was observed. The patients average stress level can be seen over a longer time period superimposed onto the above Figure 2. This raises the possibility of integrating consumer wearable analytics into the machine learning methodology developed at King's College thereby refining the diagnostic model.

\section{Aetiology}

The reasons why some patients are susceptible to a loss of smell after viral infection is unclear ${ }^{(9)}$ and the precise mechanism of olfactory loss remains elusive ${ }^{(10)}$. However, two main theories exist, conductive and sensorineural. A conductive loss has also been described as the olfactory cleft syndrome and includes inflammation and obstruction impeding transport. Sensorineural causes include damage or death of sensory cells which could also, confusingly, be caused by their inflammation. Respiratory viruses cause inflammation and resulting oedema of the nasal mucosa, possibly leading to cellular destruction. A history of fluctuating olfactory loss favours a conductive aetiology ${ }^{(2)}$ such as mucosal swelling of the olfactory cleft, which results in a conductive post viral olfactory loss ${ }^{(11)}$. 


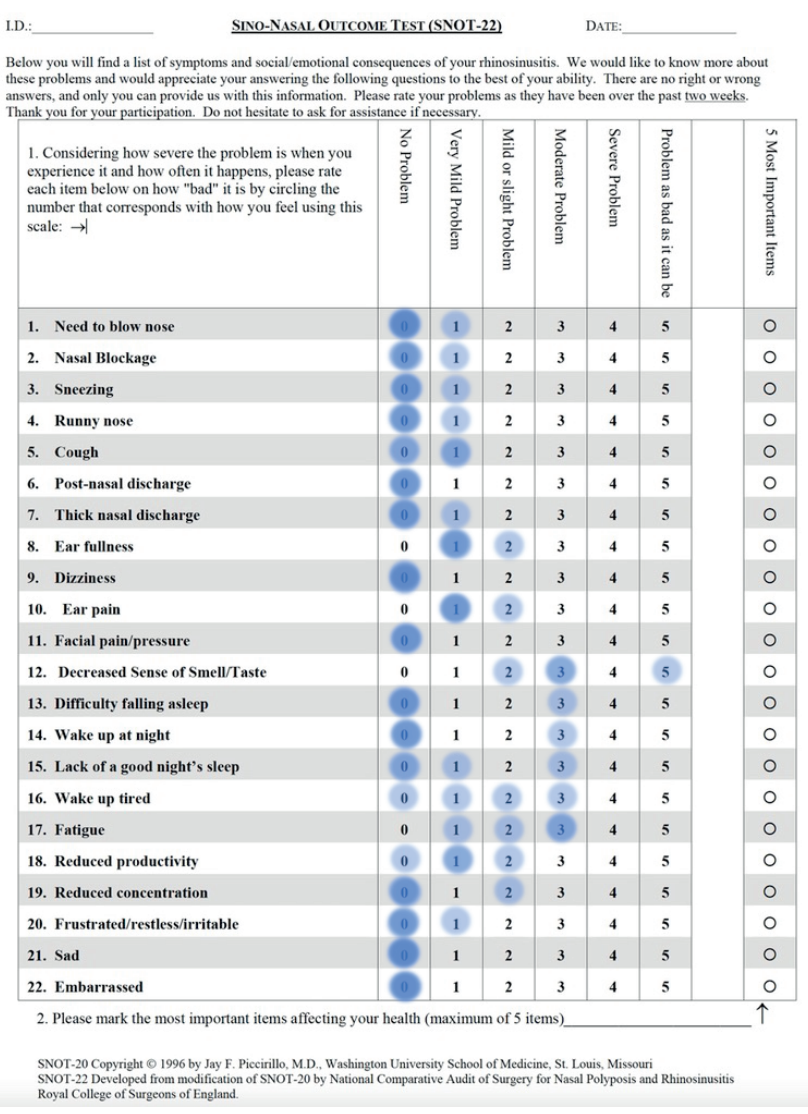

Figure 1. Sino-nasal Outcome Test (SNOT-22).

Current techniques for detecting olfactory loss can reliably determine the degree of loss but cannot distinguish between conductive or sensorineural aetiology. A variety of quantitative psychophysical tests ${ }^{(12)}$ are available to quantify changes in olfaction.

Viral infections cause varying degrees of olfactory epithelial destruction. The sensory neurones seem to be selectively affected with preservation of supporting cells. Where damage or death of olfactory receptor cells occur, regeneration should occur and may explain the transient sensory loss. Recovery is by no means guaranteed, some will have a persistent smell dysfunction. Where smell does return it will often return within 3 weeks ${ }^{(13)}$.

There are no established treatments available at this time to enhance olfactory epithelial regeneration although a number of novel therapies show promise and have been proposed.

\section{Tracking}

A variety of different software platforms have been rapidly developed and are providing insights not previously available regarding both COVID-19, smell and taste. One of the most successful has been the COVID symptom tracker app ${ }^{(14)}$ developed by King's College, London in association with Guy's and St.Thomas'
NHS Foundation trust, NIHR Biomedical Research Centre and a healthcare start-up (ZOE Global Ltd). The American Academy of Otolaryngology has developed the COVID-19 Anosmia Reporting Tool ${ }^{(15)}$ and SmellTracker ${ }^{(16)}$ are others.

The first author used the Nasal Obstructive Symptom Evaluation ${ }^{(17)}$ (NOSE) score, to document obstructive symptoms and the Sino-nasal Outcome Test (SNOT-22) (3) questionnaire to document symptoms as he developed anosmia. Although not validated for this purpose, insights can be gained regarding the COVID-19 related anosmia from a heat map image of sequential SNOT-22 scores (Figure 1).

Wearable technologies Including Garmin, WHOOP and EliteHRV ${ }^{(18)}$, may provide physiological insights into asymptomatic carriers of COVID-19. Heart rate variability is regulated by the autonomic nervous system and is a non-invasive marker of autonomic nervous system activity. It can predict stress, viral infection, myocarditis and recovery in critically ill patients. WHOOP reported ${ }^{(19)} 5$ out of 8 customers who tested positive for $\mathrm{CO}$ VID-19 showed a decrease in heart rate variability (a marker of increased stress) of $30 \%$ or more compared to a recent baseline.

\section{Treatments}

There has been no study to date about the effect of immediate treatment of post viral olfactory loss ${ }^{(11)}$. It is unknown whether high dose steroids and antiviral medication would be beneficial following the viral insult as patients rarely present early on ${ }^{(9)}$.

\section{Smell training}

It is considered as the most promising non-invasive therapeutic option for various types of smell loss although these changes might also reflect a regression to the mean.

A number of studies have suggested that smell training, i.e. sniffing four different odours; often one flowery, fruity, spicy and resinous (twice a day for 4 to 6 months) is an easy self-driven therapy with no significant side-effects. A meta-analysis showed the strong and statistically significant positive benefit of olfactory training ${ }^{(20)}$. A misunderstanding prevalent among those smell training is that if there is no perception after sniffing, there is no point in the exercise. The action of sniffing and smelling appear to activate regions of the cerebellum and may contribute to the recovery process. The development of the olfactory training ball (21) appears to provide better adherence to the training process. In those who are recovering, it can take time to receive and consider a smell. Patients should be encouraged not to dismiss smells if they cannot initially recognise them. All these comments are based on what we know about smell training and post-viral smell loss pre COVID-19. It remains to be seen whether they are applicable to COVID-19 patients. 


\section{Stress Details (?)}

Your stress level was 46 out of 100 .
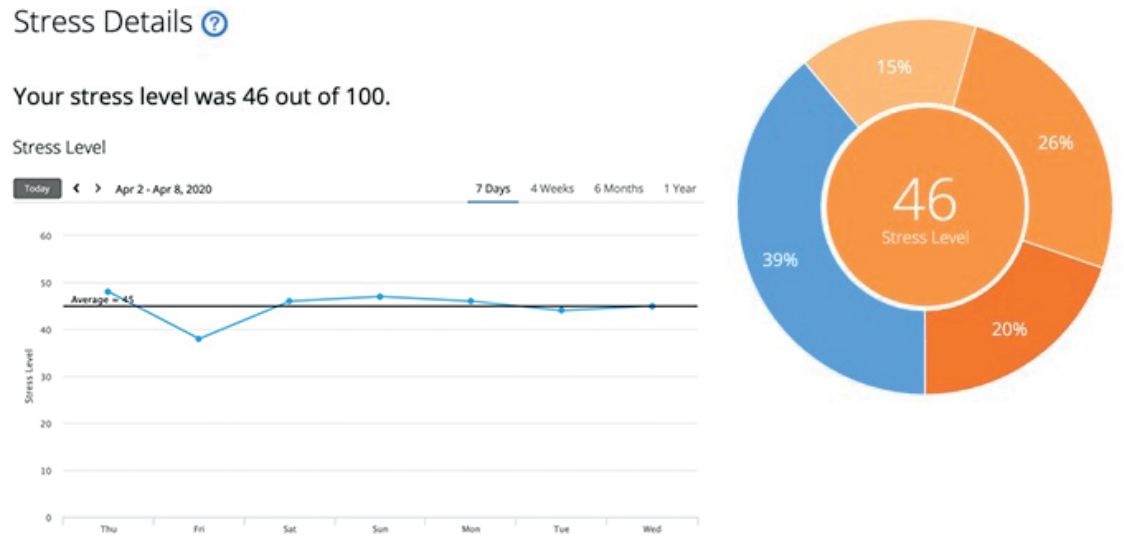

7h $24 \mathrm{~min}$

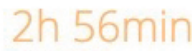

Low Stress

4h $55 \mathrm{~min}$

Medium Stress

\section{3h $45 \mathrm{~min}$}

High Stress

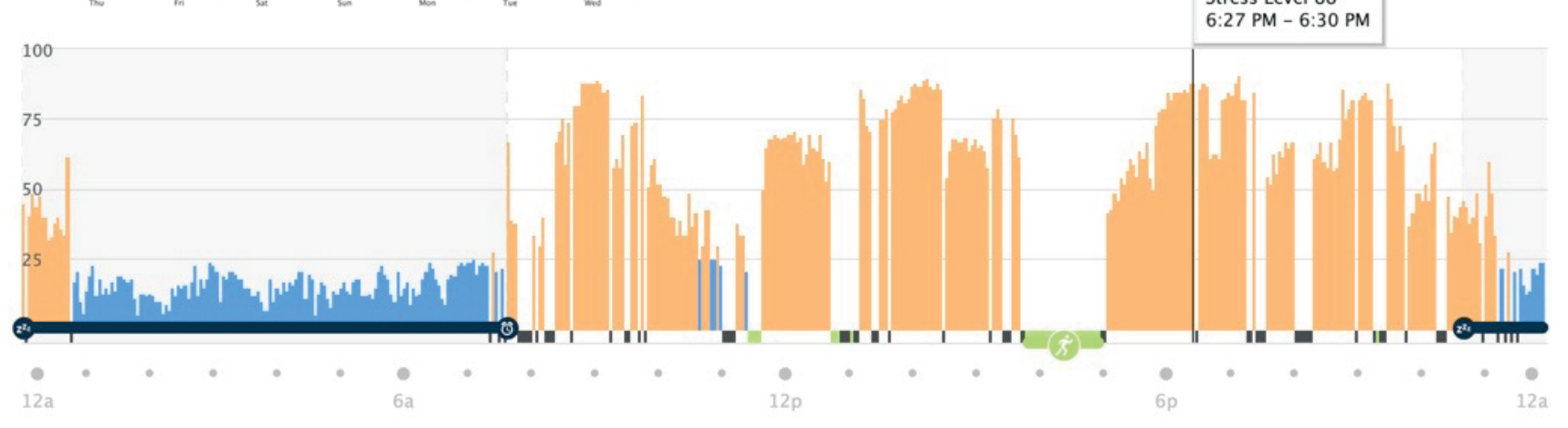

Rest Stress Unmeasurable Sleep Activity

Figure 2. Stress details.

The use of bimodal odorants, stimulating olfaction and the trigeminal shows promise ${ }^{(22)}$. Several studies have found changes in central processing of trigeminal stimuli after olfactory loss, reflecting the close interrelationship of these two sensory systems. Loss of sense of smell also leads to changes in the processing of trigeminal stimuli ${ }^{(20)}$. Of note, the first author noticed an increase in tolerance to Capsaicin following the onset of anosmia.

\section{Smell training and corticosteroids}

A randomised controlled trial, adding budesonide irrigation to smell training, significantly improved olfactory ability compared with olfactory training plus saline irrigation ${ }^{(24)}$. A randomised controlled trial placing Gelfoam dressing at the olfactory cleft, with or without local administration of $10 \mathrm{mg}$ triamcinolone showed that triamcinolone helped restore olfactory function after sinus surgery ${ }^{(25)}$.

\section{Oral and topical corticosteroids}

A brief course of oral steroid therapy can aid in determining whether inflammation (causing conductive loss) is the major determining factor of olfactory dysfunction but long term systemic steroid therapy is ill advised and may contribute to immunosuppression during active COVID-19 which we could not recommend.

\section{Omega-3}

Omega-3 has anti-inflammatory properties and promotes neuroprotection. A multi-institutional, prospective, randomised controlled trial ${ }^{(26)}$ compared nasal saline irrigation to nasal saline irrigation with $2000 \mathrm{mg}$ omega-3 supplementation following endoscopic resection of sellar or parasellar tumours. At 3 and 6 months, patients who had receiving omega-3 demonstrated significantly less persistent olfactory loss compared to patients without supplementation. After controlling for multiple confounding variables, omega-3 supplementation was found to be protective against olfactory loss. Although not directly comparable to post viral olfactory loss, this study may provide a possible area of future research and an ongoing trial of omega-3 in ICU is currently underway to treat COVID-19.

\section{Alpha-lipoic acid \\ $\alpha$-Lipoic acid (ALA) has been used to treat diabetic neuropathies with some effectiveness at higher concentrations of $600 \mathrm{mg} /$ day. In a nonblinded trial with no controls, the a range of effects of ALA on the olfactory function of 23 patients suffering from post-viral anosmia was demonstrated ${ }^{(27)}$. No other study has supported this study's findings.}


Nasal probiotic

Oral probiotics have been shown to provide a modest but significant effect on the innate inflammatory response and on decreasing viral shedding and viral load in nasal washes following infection ${ }^{(28)}$. Intranasal administration of probiotics (such as PROBIORINSE ${ }^{\mathrm{TM}}$ ) is a future area of research with regard to protecting olfaction ${ }^{(29)}$.

\section{Prognosis /Recovery}

Cain reported olfactory loss in $18.6 \%$ of patients after viral URTI ${ }^{(30)}$. Very few studies have looked at the prognosis of post viral olfactory dysfunction ${ }^{(31)}$. Unexpectedly, $86 \%$ of patients with post viral olfactory loss reported subjective improvement and UPSIT scores improved in $90 \%{ }^{(31)}$. Recovery to normosmia was most frequent in patients with mild to moderate hyposmia. Jafek et al. reported that recovery of smell should occur within 3 weeks ${ }^{(13)}$. The first author repeated olfactory testing 6 weeks after total anosmia was noted. Smell had returned to normosmia with a UPSIT score of 39 within the 90th percentile. Although a case report it provides evidence that smell function can return to expected levels for age after the olfactory system has received an insult from COVID-19.

\section{Conclusions and recommendations}

A loss of taste and smell as well as autonomic dysfunction appear to be early warning markers of COVID-19 infection ${ }^{(32)}$, but fever and coughing are frequently also present.

Loss of sense of smell and taste, when combined with other symptoms, continues to make it likely that an individual has contracted COVID-19.

Wearable consumer technology that records HRV may provide an early non-invasive diagnosis of viral infections in advance of the loss of sense of smell.
Recovery from anosmia is likely to begin within 3 weeks of initial loss and can recover completely to within normal age adjusted ranges within 6 weeks. Smell training with bimodal odorants (22) (stimulating both olfaction and trigeminal systems) with the addition of budesonide irrigation appears to offer the most promise for future research.

Omega-3 supplementation may confer an additional benefit and the contribution of topical steroids placed within the nose such as PROPEL ${ }^{\mathrm{TM}} \&$ SINUVA ${ }^{\oplus}$ as early adjunctive therapy to aid recovery should be studied though randomised controlled trials.

\section{Acknowledgments}

None.

\section{Authorship contribution}

DW wrote the manuscript and provided case data. CK provided data regarding smell training and edited the manuscript, NA edited the manuscript.

\section{Conflict of interest}

CK is the founder of AbScent, a charity registered in England and Wales No. 1183468, that supports people with smell loss.

\section{Ethics approval and consent to participate}

Not applicable.

\section{Consent for publication}

Not applicable.

\section{Availability of data and materials}

Not applicable.

\section{Funding}

Not applicable.

\section{References}

1. Lechner M, Chandrasekharan D, Jumani K, et al. Anosmia as a presenting symptom of SARS-CoV-2 infection in healthcare workers - A systematic review of the literature, case series, and recommendations for clinical assessment and management. Rhinology. Published online May 2020. doi:10.4193/ Rhin20.189

2. Seiden AM, Duncan HJ. The diagnosis of a conductive olfactory loss. Laryngoscope. 2001;111(1):9-14.

3. Hopkins C, Gillett S, Slack R, Lund VJ, Browne JP. Psychometric validity of the 22-item Sinonasal Outcome Test. Clin Otolaryngol. 2009;34(5):447-454.

4. Ottaviano G, Carecchio M, Scarpa B, Marchese-Ragona R. Olfactory and rhinological evaluations in SARS-CoV-2 patients complaining of olfactory loss. Rhinology.
Published online April 2020. doi:10.4193/ Rhin20.136

5. Doty RL, Shaman P, Kimmelman CP, Dann MS. University of pennsylvania smell identification test: A rapid quantitative olfactory function test for the clinic. Laryngoscope. 1984;94(2):176-178.

6. Chen WL, Kuo CD. Characteristics of Heart Rate Variability Can Predict Impending Septic Shock in Emergency Department Patients with Sepsis. Acad Emerg Med. 2007;14(5):392-397.

7. Griffin MP, Moorman JR. Toward the early diagnosis of neonatal sepsis and sepsislike illness using novel heart rate analysis. Pediatrics. 2001;107(1):97-104.

8. Carter R, Hinojosa-Laborde C, Convertino VA. Heart rate variability in patients being treated for dengue viral infection: New insights from mathematical correc- tion of heart rate. Front Physiol. 2014;5 FEB(February):1-5.

9. Seiden AM. Postviral olfactory loss. Otolaryngol Clin North Am. 2004;37(6 SPEC. ISS.):1159-1166.

10. Heidari F, Karimi E, Firouzifar $M$, et al. Anosmia as a prominent symptom of COVID-19 infection. Rhinology. Published online April 2020. doi:10.4193/Rhin20.140

11. Seo BS, Lee HJ, Mo J-H, Lee CH, Rhee C-S, Kim J-W. Treatment of Postviral Olfactory Loss With Glucocorticoids, Ginkgo biloba, and Mometasone Nasal Spray. Arch Otolaryngol Neck Surg. 2009;135(10):1000.

12. Hummel T, Sekinger B, Wolf SR, Pauli E, Kobal G. "Sniffin" sticks'. Olfactory performance assessed by the combined testing of odor identification, odor discrimination and olfactory threshold. Chem Senses. 1997;22(1):39-52. 
13. Jafek BW, Hartman D, Eller PM, Johnson EW Strahan RC, Moran DT. Postviral Olfactory Dysfunction. Am J Rhinol. 2007;4(3):91-100.

14. COVID Symptom Study - Help slow the spread of COVID-19. Accessed May 19, 2020. https://covid.joinzoe.com/

15. COVID-19 Anosmia Reporting Tool | American Academy of OtolaryngologyHead and Neck Surgery. Accessed May 19, 2020. https://www.entnet.org/content/ reporting-tool-patients-anosmia-relatedcovid-19

16. SmellTracker. Accessed May 19, 2020 https://smelltracker.org/take-test/yoursense-smell

17. Stewart MG, Witsell DL, Smith $T L$, et al. Development and validation of the Nasa Obstruction Symptom Evaluation (NOSE) Scale. Otolaryngol Head Neck Surg. 2004 Feb;130(2):157-63.

18. Elite HRV - Top Heart Rate Variability App, Monitors, and Training. Accessed May 19, 2020. https://elitehrv.com/

19. Heart Rate \& Valuable WHOOP Data in COVID-19 | Coronavirus | WHOOP. Accessed May 19, 2020. https://www.whoop.com/ thelocker/whoop-data-coronavirus/

20. Sorokowska A, Drechsler E, Karwowski M, Hummel T. Effects of olfactory training: A meta-analysis. Rhinology. 2017;55(1):17-26.

21. Saatci O, Altundag A, Arici O, Thomas D. Olfactory training ball improves adherence and olfactory outcomes in post infectious olfactory dysfunction. Eur Arch Otorhinolaryngol. 2020 Apr 3. doi: 10.1007/ s00405-020-05939-3.

22. Poletti SC, Michel E, Hummel T. Olfactory training using heavy and light weight molecule odors. Perception. 2017;46(3-4):343 351.

23. Reichert JL, Schöpf V. Olfactory Loss and Regain: Lessons for Neuroplasticity. Neuroscientist. 2018;24(1):22-35.

24. Nguyen TP, Patel ZM. Budesonide irrigation with olfactory training improves outcomes compared with olfactory training alone in patients with olfactory loss. Int Forum Allergy Rhinol. 2018;8(9):977-981.

25. Bardaranfar $\mathbf{M H}$, Ranjbar Z, Dadgarnia $\mathbf{M H}$ et al. The effect of an absorbable gelatin dressing impregnated with triamcinolone within the olfactory cleft on polypoid rhinosinusitis smell disorders. Am J Rhinol Allergy. 2014;28(2):172-175.

26. Yan $\mathrm{CH}$, Rathor A, Krook K, et al. Effect of Omega-3 Supplementation in Patients With Smell Dysfunction Following Endoscopic Sellar and Parasellar Tumor Resection: A Multicenter Prospective Randomized Controlled Trial. Neurosurgery. 2020;0(0):1-8.

27. Hummel T, Heilmann S, Hüttenbriuk KB Lipoic acid in the treatment of smell dysfunction following viral infection of the upper respiratory tract. Laryngoscope. 2002;112(11):2076-2080.

28. Turner RB, Woodfolk JA, Borish L, et al. Effect of probiotic on innate inflammatory response and viral shedding in experimental rhinovirus infection - A randomised controlled trial. Benef Microbes. 2017:8(2):207-
215.

29. Lehtinen MJ, Hibberd AA, Männikkö S, et al. Nasal microbiota clusters associate with inflammatory response, viral load, and symptom severity in experimental rhinovirus challenge. Sci Rep. 2018;8(1):1-12.

30. Cain WS, Gent JF, Goodspeed RB, Leonard G. Evaluation of Olfactory Dysfunction in the Connecticut Chemosensory Clinical Research Center. Laryngoscope. 1988;98(1):83???88.

31. Lee DY, Lee WH, Wee JH, Kim JW. Prognosis of postviral olfactory loss: Follow-up study for longer than one year. Am J Rhinol Allergy. 2014;28(5):419-422.

32. Hopkins C, Surda P, Kumar N. Presentation of new onset anosmia during the COVID19 pandemic. Rhinology. Published online April 2020. doi:10.4193/Rhin20.116

David E.J. Whitehead

Department of Otorhinolaryngology

James Cook University Hospital

Middlesbrough, TS4 3BW

United Kingdom

E-mail: david@ent.org.uk

ISSN: 2589-5613 / ( 2020 The Author(s). This work is licensed under a Creative Commons Attribution 4.0 International License. The images or other third party material in this article are included in the article's Creative Commons license, unless indicated otherwise in the credit line; if the material is not included under the Creative Commons license, users will need to obtain permission from the license holder to reproduce the material. To view a copy of this license, visit http://creativecommons.org/ licenses/by/4.0/ 